\title{
ИСПОЛЬЗОВАНИЕ РЫНОЧНЫХ ОЖИДАНИЙ В УПРАВЛЕНИИ РОССИЙСКИМИ КОМПАНИЯМИ
}

Ивашковская И.В. ${ }^{1}$, Дьяченко К.В. ${ }^{2}$

\begin{abstract}
На развитых рынках менеджеры стремятся представить инвесторам большие массивы информации, чтобы минимизировать разрыв между фундаментальной и рыночной стоимостями. Данные корректировки со стороны менеджеров называют управлением ожиданиями. В академической литературе рассматриваются два способа моделирования рыночных ожиданий: через модели временных рядов и через прогнозы аналитиков. В данном исследовании применяется второй способ. Именно аналитики формируют информационное пространство, которое, в свою очередь, влияет на решения инвесторов и, соответственно, рыночные курсы акций компаний.
\end{abstract}

Применяется ли управление ожиданиями компаниями на российском рынке? Каким образом происходит взаимодействие менеджеров и аналитиков в рамках управления ожиданиями? От каких характеристик компании зависит ее склонность к применению управления ожиданиями? Данная статья обобщает модель и результаты только первого этапа исследования - выявления наличия управления ожиданиями на российском рынке капитала. Эмпирический анализ механизма взаимодействия менеджеров и аналитиков, а также результаты исследования нами характеристик компаний, склонных применять управление ожиданиями, публикуются во второй части в 2014 году.

В первом параграфе рассматриваются ключевые исследования в выявлении фактов управления ожиданиями. Во втором параграфе представлена модель исследования. В третьем параграфе обоснованы гипотезы. Характеристики выборки компаний и необходимые переменные показаны в четвертом параграфе. Оценки модели и анализ полученных результатов даны в пятом параграфе.

\section{Управление рыночными ожиданиями: результаты исследований}

Феномен управления рыночными ожиданиями получил наибольшее распространение на развитых рынках, что связано с особенностями законодательства и относительно высокой степенью эффективности рынка капитала. Применение данного инструмента нарастало в конце 1990-х (Bartov, Brown, 2002; Matsumoto, 2002) и приобрело наибольшую популярность в начале 2000-х после ряда скандалов, связанных с раскрытием информации (Koh et al., 2008). Важная причина управления ожиданиями - стремление получить рост доходности акций в случае превышения ожиданий инвесторов по доходности. В работе Грэхэм (Graham et al., 2004) на данных опроса более 400 финансовых директоров и интервью с генеральными директорами компаний из разных отраслей выявлено, что такая цель реально ставится с компаниях. Если фирма оправдывает рыночные ожидания, то доходность ее акций растет (Bartov et al., 2002). Выявлен также факт асимметричности рыночных реакций на оправданность и неоправданность фирмой ожиданий. Рынок сильнее реагирует, если фирма не оправдала ожидания. Исследования показывают, что после скандалов начала 2000-х произошли некоторые изменения в размере рыночной премии за оправданные ожидания. До начала 2000-х рыночную премию получали все фирмы, превысившие ожидания, даже если это превышение было ниже одного цента на акцию. Впоследствии рынок стал скептично относиться к факту оправдания ожиданий (Koh et al., 2008). Премия для компаний, превысивших ожидания по прибыли менее чем на 1 цент на акцию, исчезла. Премия для компаний, превысивших ожидания по прибыли более чем на 1 цент на акцию, уменьшилась. Также сократилось и наказание за неоправданные

1. Д-р эконом. наук, ординарный профессор, руководитель академического департамента финансов, заведующая исследовательской лаборатории корпоративных финансов НИУ ВШЭ. 
ожидания. В общем и целом, чтобы получить размер премии, существовавший до скандала, менеджерам стало необходимо превышать ожидания в большей степени.

Вторая причина заключается в том, что оправданные ожидания способствуют стабилизации курса акций компании, снижению волатильности и, как следствие, - снижению затрат на капитал. В-третьих, неоправданные ожидания интерпретируются многими инвесторами как сигнал о серьезных проблемах в компании, низкой эффективности менеджмента, что помимо падения курса акций влечет за собой множественные объяснения со стороны менеджеров перед аналитиками и журналистами. Время, потраченное на подобные объяснения, является вторым по значимости типом экономических потерь от неоправданных ожиданий (Graham et al., 2005).

Анализ издержек неоправданных ожиданий показывает, что пересмотр ожиданий в сторону снижения влияет на доходность акций. Выявлено, что изменения в ожиданиях объясняют около $45 \%$ дисперсии доходности акций, причем регрессионные коэффициенты при изменениях в ожиданиях положительны (Copeland et al., 2004). Это означает, что пересмотр аналитиками своих прогнозов в сторону занижения негативно сказывается на доходности акций компании. Другими словами, данное снижение доходности как раз и является издержками применения управления ожиданиями. Однако в работе Мацумото (Matsumoto, 2002) показано, что убыток от неоправданных ожиданий гораздо выше, чем убыток от их пересмотра в сторону занижения. Управлять ожиданиями и отвечать им выгоднее, чем не оправдывать ожидания. Поэтому политика управления ожиданиями стала становиться важным элементом управления6 ориентированного на приращение стоимости компании (Ивашковская, 2009).

Другой вид издержек управления ожиданиями - это недополученная доходность. Если фирма манипулировала ожиданиями аналитиков, то, оправдав ожидания, она получает более низкую доходность, чем те компании, которые оправдали ожидания без аналогичных манипуляций. Существует дисконт к доходности акций тех фирм, которые оправдали ожидания путем различного рода манипуляций (Athanasakou et al., 2011). Однако такие компании все равно получают рыночную премию к доходности по сравнению с компаниями, которые не оправдали рыночных ожиданий.

Исследователи отмечают конфликт интересов, возникающий как в среде менеджеров, так и в среде аналитиков (Bradshaw, 2011). Первым и самым серьезным источником конфликта является возможность получения денежного вознаграждения от инвестиционных банков в случае составления желаемого менеджерами и банками прогноза по сделкам с компаниями. Тогда при анализе качества и последствий совершаемых сделок аналитики склонны изменять свои прогнозы в сторону, угодную компаниям. Вторым источником конфликта является стремление аналитиков к сотрудничеству с менеджерами. Такое сотрудничество позволяет аналитикам иметь более широкий доступ к информации о компании, получаемой через общение с менеджерами. На прогнозы аналитиков сильно влияет состав инвестиционных портфелей их клиентов: о бумагах, находящихся в портфеле инвестора, говорят либо хорошо, либо ничего (Bradshaw, 2011). В связи с этим прогнозы аналитиков являются неполными и смещенными. Кроме того, источником конфликта является ситуация, когда компания платит аналитикам, чтобы они делали прогнозы относительно ее положения на рынке. Перечисленные факторы сводятся к тому, что та или иная сторона манипулирует ожиданиями аналитиков в свою пользу. Наконец, существует источник конфликта, основанный на природе самого аналитика. Считается, что часто аналитики переоценивают или недооценивают определенную информацию, в связи с чем их прогнозы неточно отражают реальное положение дел.

Управление ожиданиями является широко изучаемым феноменом на развитых рынках, но существует очень мало аналогичных исследований на развивающихся рынках. Тем не менее, как показано в исследовании Браун и Хиггинс (Brown, Higgins, 2005), управление ожиданиями начинает применяться и на развивающихся рынках. Подобные исследования на данных развивающихся рынков капитала помогут более глубоко понять природу данного феномена, а также сделать выводы о его универсальности. 


\section{Модель исследования: выявление управления рыночными ожиданиями со стороны менеджеров на российском рынке}

Цель первого этапа исследования - определить, существует ли на российском рынке управление ожиданиями со стороны менеджеров компаний. В качестве прокси для рыночных ожиданий взят консенсус-прогноз всех аналитиков, покрывающих компанию. Консенсус-прогноз усредняет и поэтому нивелирует субъективизм каждого отдельного аналитика, что дает возможность использовать его как прокси-переменную для рыночных ожиданий. Важно отметить, что под рыночными ожиданиями далее во всей работе подразумеваются прогнозы аналитиков по размеру прибыли на акцию компании.

Первая группа методов выявления управления ожиданиями заключается в анализе заявлений и публикации ожидаемых результатов со стороны самих менеджеров компаний (Cotter et al., 2006). Необходим анализ информации о времени и содержании публичных заявлений менеджеров об их ожиданиях относительно результатов деятельности компании, время и тип реакции аналитиков на данные заявления. Однако такой тип данных на российском рынке является доступным для очень малой выборки компаний, поэтому использована вторая группа методов. Вторая группа объединяет способы анализа последствий управления ожиданиями: динамики прогнозов аналитиков в течение периода, поведения курсов акций после публикации отчетности, размера и распределения ошибки прогноза, изменений в прибыли компании и других показателей (Matsumoto, 2002; Sankaraguruswamy, Sweeney, 2005).

В данном исследовании применены три способа проверки существования управления ожиданиями на российском рынке: графический анализ, анализ сопряженности признаков, тестирование рациональности ожиданий аналитиков. Первый способ - использование графического анализа в оценке динамики двух показателей: частоты, с которой фирмы оправдывают ожидания, и частоты, с которой фирмы показывают положительный прирост прибыли по сравнению с прошлым годом (Matsumoto, 2002). Показатели измеряются отдельно для каждого года и считаются следующим образом. Первый показатель равен сумме фирм, которые оправдали ожидания в текущем году, разделенной на общее количество фирм в выборке. Второй показатель равен сумме фирм, которые показали положительную динамику прибыли в текущем году по сравнению с прошлым годом, разделенной на общее количество фирм в выборке. Оба показателя измеряются в процентах. Этот способ, по сути, - экспресс-тест. Если связи между динамикой двух показателей не наблюдается, то можно сделать вывод, что фирмы оправдывают или не оправдывают ожидания по каким-то иным причинам, включая управление ожиданиями аналитиков.

Второй способ основан на анализе сопряженности признаков (Matsumoto, 2002; Brown, Higgins ,2005). Необходимо без применения регрессионного анализа выявить возможную связь между показателями управления со стороны менеджеров и динамикой прибыли, пересмотром аналитиками ожиданий, фактом оправдания ожиданий. Такой анализ помогает сопоставить различные последствия управления ожиданиями с предполагаемыми причинами и выяснить, являются ли причины и последствия связанными друг с другом. В целях такого анализа были созданы следующие бинарные переменные, отвечающие за предполагаемые причины изменений в динамике прибыли, поведении менеджеров и аналитиков. Первая переменная отражает факт того, оправдала ли компания ожидания, носит название MEET и определяется следующим образом:

$\mathrm{MEET}=\left\{\begin{array}{l}1, \text { если фирма оправдывает или превышает ожидания, } \\ 0, \text { в любом другом случае }\end{array}\right.$

Вторая переменная отслеживает, осуществляла ли фирма управление ожиданиями или нет. Эта переменная задана двумя способами, которые основываются на разных критериях выявления управления ожиданиями. Данная переменная, следуя терминологии Браун и Хиггинс (Brown, Higgins, 2005), названа MGUI. В случае управления ожиданиями со стороны 
менеджеров аналитики делают два типа прогнозов: на основе всей имеющейся информации на рынке, (назовем его ожидаемым) и созданный с учетом новой информации, полученной от менеджеров и призванной скорректировать ожидания аналитиков. Именно второй тип прогноза публикуется аналитиками в конце периода, и относительно него оценивается уровень публикуемой прибыли. Соответственно, если публикуемый прогноз оказывается ниже ожидаемого, то существует какая-то причина, по которой аналитики опубликовали прогноз, заведомо не совпадающий с реальными показателями. Иными словами, на рынке существует управление ожиданиями. В противном случае, если публикуемый и ожидаемый прогнозы совпадают, то это означает, что менеджеры не манипулируют ожиданиями аналитиков, либо аналитики не учитывают новую информацию от менеджеров в своих прогнозах, то есть не поддаются управлению. Поэтому необходимо оценить разницу между публикуемым и ожидаемым прогнозами. Данная разница оценивается следующим образом.

$$
U E F_{i j t}=C F_{i j t}-E\left[F_{i j t}\right]
$$

В уравнении (2) индекс $i$ означает фирму, $j$ - отрасль, $t$ - год; $C F$ - это публикуемый аналитиками прогноз (consensus forecas; $E[F]$ - ожидаемый прогноз, который аналитики должны были опубликовать.

Ожидаемый прогноз является ненаблюдаемой величиной, поэтому его необходимо оценить, например, при помощи модели случайного блуждания (Matsumoto, 2002). Другими словами, размер прибыли на акцию в текущем периоде моделируется следующим образом.

$E P S_{i j t}=E P S_{i j, t-1}+D_{i j t}$

где $E P S_{i j t}$ - это размер прибыли на акцию в текущем периоде, $E P S_{i j, t-1}$ - размер прибыли на акцию в прошлом периоде, $D_{i j t}$ - соответствующая разница в этих двух значениях, то есть:

$D_{i j t}=E P S_{i j t}-E P S_{i j, t-1}$

Прогноз по прибыли на акцию в конце текущего периода равен математическому ожиданию прибыли. Данное математическое ожидание учитывает всю информацию, доступную аналитикам, поэтому является ожидаемым прогнозом, то есть . Поэтому ожидаемый прогноз моделируется согласно уравнению (5).

Последним этапом оценки является моделирование ожидаемой ошибки прогноза. Данная ошибка рассчитывается посредством уравнения (6).

$$
E\left[D_{i j t}\right]=\alpha_{i t}+\beta_{i t} *\left(\frac{D_{i j, t-1}}{P_{i j, t-2}}\right) * P_{i j, t-1}
$$

Уравнение (6) является регрессией, оцененной методом OLS. В данной регрессии независимая переменная $\mathrm{P}$ - это курс акций компании на конец указанного в индексе периода. Порядок оценки перечисленных уравнений следующий: (4), (6), (5), (2).

Далее необходимо понять, является ли публикуемый прогноз ниже ожидаемого, поэтому была введена бинарная переменная DOWN, которая задается формулой (7).

$D O W N=\left\{\begin{array}{l}1, \text { если } E P S_{i j t}<0 \\ 0, \text { в любом другом случае }\end{array}\right.$

В итоге на основе уравнений (1)-(6) была посчитана переменная MGUI, отвечающая за факт осуществления управления ожиданиями со стороны менеджеров. Смысл этой переменной заключается в том, что аналитики пересмотрели свой прогноз в сторону понижения, и это позволило менеджерам оправдать ожидания по прибыли.

$M G I U=\left\{\begin{array}{l}1, \text { если } D O W N=1 \text { и } M E E T=1 \\ 0, \text { в любом другом случае }\end{array}\right.$ 
Второй способ основан на обобщении всех последствий управления ожиданиями в одну бинарную переменную, названную BGUI ${ }^{1}$ (Bartov et al., 2002). Авторы подчеркивают, что в начале периода аналитики публикуют один прогноз, однако в случае управления со стороны менеджеров они пересматривают его и публикуют новый прогноз в конце периода до даты публикации отчетности. Основываясь на мотивации менеджеров занизить прогнозы аналитиков, чтобы оправдать их, авторы предполагают, что конечный прогноз должен быть ниже начального. Более того, реальная прибыль должна быть выше конечного прогноза, но ниже начального. Первое условие гарантирует факт оправдания менеджерами ожиданий. Второе условие определяет мотив менеджеров занизить ожидания аналитиков. Переменная BGUI задается следующим образом:

$$
B G U I=\left\{\begin{array}{l}
\text { если (1) реальная прибыль на акцию меньше начального прогноза } \\
\text { (2) конечный прогноз по прибыли ниже начального прогноза } \\
\text { (3) реальная прибыль выше конечного прогноза } \\
0 \text { в любом другом случае }
\end{array}\right.
$$

Среди четырех перечисленных бинарных переменных BGUI была принята за основную, MGUI, MEET и DOWN используются как дополнительные переменные для проверки устойчивости результатов. Такое решение было принято по ряду причин. Во-первых, структура переменной BGUI является более прозрачной и простой, чем у MGUI, что позволяет делать меньше предположений относительно поведения аналитиков. Такая простота предполагает уменьшение количества ошибок спецификации переменной. Во-вторых, при построении BGUI не используются лаговые значения показателей, что позволяет сохранить размер выборки. В-третьих, данная переменная является более полной, чем MEET и DOWN, так как учитывает все последствия управления ожиданиями на рынке.

В рамках анализа сопряженности признаков указанные переменные были скомбинированы со следующими признаками: оправдала ли компания ожидания аналитиков или нет, показала ли компания в текущем периоде прибыли или, напротив, убытки. Анализ сопряженности поможет понять, существует ли связь между предполагаемыми причинами и данными последствиями, то есть имеет ли место управление ожиданиями на рынке.

Третий способ выявления управления ожиданиями состоит в тестировании прогнозов аналитиков на рациональность и основывается на предположении, что в случае отсутствия вмешательства со стороны менеджеров аналитики формируют рациональные прогнозы. Другими словами, ожидаемое значение ошибки прогноза равно нулю. Математически это предположение можно выразить при помощи ряда формул. Прогноз аналитика о размере прибыли на акцию в конце текущего периода выражается формулой 10:

$$
F\left[E P S_{t}\right]=E P S_{t}-\varepsilon_{t},
$$

где $F\left[E P S_{t}\right]$ - это прогноз по прибыли на текущий период, $E P S_{t}$-фактическая прибыль на акцию на конец текущего периода, $\varepsilon_{t}$-ошибка прогноза. Следовательно,

$\varepsilon_{t}=E P S_{t}-F\left[E P S_{t}\right]$

Согласно приведенному определению рациональности ожиданий, имеем следующее равенство:

$$
E\left[\varepsilon_{t}\right]=E\left[E P S_{t}-F\left[E P S_{t}\right]\right]=0
$$

Таким образом, в рамках рассматриваемой модели предполагается следующее распределение ошибки прогноза:

$$
\varepsilon_{t} \sim N\left(0, \sigma_{\varepsilon}^{2}\right)
$$

Нормальное распределение ошибки является предположением, сделанным для того, чтобы обеспечить выполнение условий теоремы Гаусса-Маркова.

1. Название переменной взято из работы Brown, L., Higgins, Н. (2005). Managers' forecast guidance of analysts: International evidence, Journal of accounting and Public Policy, 24 (2005) 280-299. 


\section{Гипотезы исследования}

Учитывая определение рациональности аналитиков, а также предположение о распределении ошибки прогноза, были проведены три различных теста. На первом этапе исследования использован тест на систематичность ошибок прогнозов аналитиков в ходе сравнительного анализа распределений ошибок начального и конечного прогнозов. В ходе теста проверяются следующие гипотезы.

Гипотеза 1.1. Первоначальный прогноз аналитиков сделан без вмешательства менеджеров и отражает реальное восприятие компании аналитиками. Ошибки начальных прогнозов имеют нормальное распределение с нулевым математическим ожиданием, то есть:

$$
\varepsilon_{t}^{\text {begin }} \sim N\left(0, \sigma_{\varepsilon^{\text {begin }}}^{2}\right)
$$

Если данная гипотеза отвергается, это означает, что начальные прогнозы аналитиков содержат систематическую ошибку, которая отражает качества самих аналитиков. Примером таких качеств могут служить наивность ожиданий аналитиков, переоценка какой-либо информации, неверность используемых моделей и т.д.

Гипотеза 1.2. Ошибки конечных прогнозов имеют нормальное распределение с нулевым математическим ожиданием. То есть:

$\varepsilon_{t}^{\text {end }} \sim N$

Если первая гипотеза не отвергается, а вторая отвергается, это означает, что изначально аналитики рациональны, однако к концу периода они пересматривают свои ожидания, делая при этом систематически ошибки. В случае, когда распределение ошибок конечных прогнозов смещено вправо, это означает, что аналитики систематически занижают свои прогнозы к концу периода, что свидетельствует об управлении ожиданиями.

$$
E P S_{t}=\alpha+\beta * F\left[E P S_{t}\right]+\varepsilon_{t}, \varepsilon_{t} \sim N\left(0 ; \sigma_{\varepsilon}^{2}\right)
$$

На втором шаге применен тест на рациональность аналитиков, заключающийся в проверке коэффициентов регрессионной модели, представленной в уравнении (16) (записано в векторной форме, поэтому у переменных присутствует только временной индекс). Гипотеза также формулируется для начальных и конечных прогнозов.

Гипотеза 2.1. В уравнении (16) для начальных прогнозов коэффициенты имеют следующие значения.

$\left\{\begin{array}{l}\alpha=0 \\ \beta=1\end{array}\right.$

Гипотеза 2.2. В уравнении (16) для конечных прогнозов коэффициенты имеют следующие значения.

$\left\{\begin{array}{l}\alpha=0 \\ \beta=1\end{array}\right.$

Если гипотезы 2.1 и 2.2 не отвергаются, это означает, что ожидаемое значение прибыли равно прогнозу, сделанному аналитиками. Другими словами, ожидания аналитиков являются рациональными для обоих прогнозов.

На третьем шаге использован тест на учет аналитиками всей доступной информации по компании. Поэтому определяется значимость коэффициентов в следующей регрессионной модели (данная модель записана в векторной форме):

$$
F\left[E P S_{t}\right]=\delta_{0}+\delta_{1} * E P S_{t-i}+\delta_{2} * \text { Error }_{t-i}+\delta_{3} * \operatorname{Return}_{i}+\varepsilon_{t}, \forall i=1,2,3 \ldots T, \varepsilon_{t} \sim N\left(0 ; \sigma_{\varepsilon}^{2}\right),
$$

где Return $_{t-i}$ - доходность акций прошлых периодов, Error ${ }_{t-i}$ - ошибка конечного прогноза прошлых периодов.

Гипотеза 3.1. Коэффициенты $\delta_{1}, \delta_{2}$ и $\delta_{3}$ в уравнении (17) для начальных прогнозов являются значимыми. 
Гипотеза 3.2. Коэффициенты $\delta_{1} u \delta_{2}$ и $\delta_{3}$ в уравнении (17) для конечных прогнозов являются значимыми.

Если гипотезы 3.1 и 3.2 не отвергаются, это значит, что аналитики используют ту информацию, которая имеется у них в распоряжении для формирования прогнозов. Вывод относительно степени рациональности аналитиков делается на основе результатов проверки всех шести гипотез. Предполагается, что гипотезы 1.1, 2.1 и 3.1 не будут отвергнуты, что означает, что начальные прогнозы являются рациональными. Гипотезы 1.2, 2.2 и 3.2 будут отвергнуты, что подтвердит предположение о нерациональности конечных ожиданий, так как они были сформированы под влиянием менеджеров. Также предполагается, что распределение ошибок конечных прогнозов имеет положительное математическое ожидание, то есть конечные прогнозы систематически занижаются.

\section{Характеристики выборки}

В качестве объекта исследования были выбраны 200 крупнейших по капитализации российских компаний, торгующихся на ММВБ. Исследуемый период составляет восемь лет: с 2005 по 2012 год, включая кризисный год, поэтому в целях детализации анализа период разделен на три части: докризисный (2005-2007 гг.), кризисный (2008 г.) и посткризисный (2009-2012 гг.). В качестве источника информации служит база данных Bloomberg. Группировка по отраслям была проведена на основе классификации Bloomberg первого уровня (диаграмма 1). Все фундаментальные показатели взяты с годовой частотой, значения консенсус-прогнозов аналитиков - с месячной. В целях учета инфляционного влияния данные используются в долларовом исчислении.

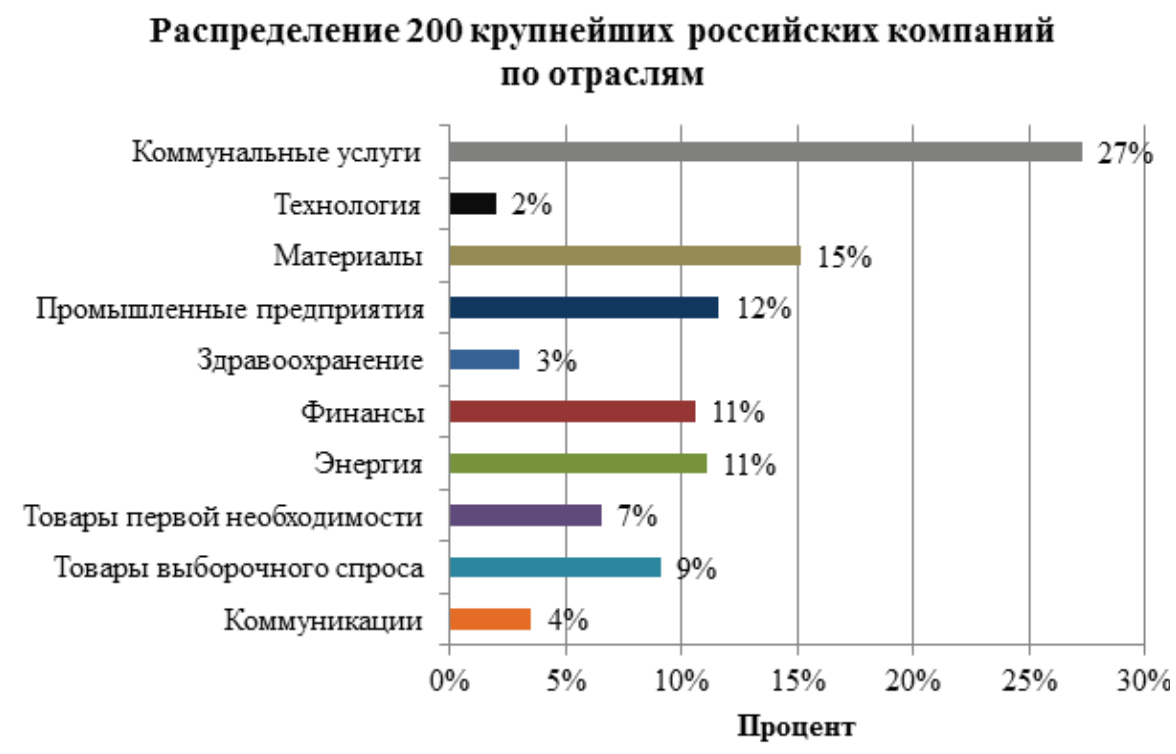

Диаграмма 1. Распределение 200 крупнейших компаний по отраслям согласно классификаџии Bloomberg первого уровня

\section{Источник: Рассчитано на основе данных Bloomberg.}

Описание исследуемых переменных приведено в таблице 1. Прогнозные показатели отражают ежемесячный пересмотр прогноза, сделанного в текущем году, на конец текущего года. Сформированы соответствующие каждому году прогнозы, сделанные в начале (первый доступный в рассматриваемом году прогноз) и в конце периода (последний доступный прогноз на рассматриваемый год). Обычно конечный прогноз выпускается в следующем после прогнозируемого года перед раскрытием годовой отчетности. В среднем по российским компаниям это март-апрель следующего года. В случае когда по компании выходил только один прогноз за год, наблюдение удалялось из выборки. 
Переменные для модели исследования

\begin{tabular}{|c|c|}
\hline Название переменной & Обозначение переменной в базе данных Bloomberg \\
\hline Чистая прибыль & is_comp_net_income_adjust \\
\hline Чистая прибыль на акцию & is_comp_eps_adjusted \\
\hline Средневзвешенные затраты на капитал & wacc \\
\hline Совокупные активы & bs_tot_assets \\
\hline Балансовая стоимость основного капитала & bs_gross_fix_asset \\
\hline Обратный мультипликатор $\mathrm{B} / \mathrm{P}^{1^{*}}$ & market_capitalization_to_bv \\
\hline Мультипликатор P/E & pe_ratio \\
\hline Бета & beta_raw_overridable \\
\hline $\begin{array}{l}\text { Прогноз прибыли на акцию компании на текущий } \\
\text { год, сделанный в текущем году }\end{array}$ & best_eps \\
\hline $\begin{array}{l}\text { Прогноз долгосрочного роста прибыли на акцию } \\
\text { компании на текущий год, сделанный в текущем } \\
\text { году }\end{array}$ & best_ltg_eps \\
\hline Цена акции & px_last \\
\hline
\end{tabular}

Источник: Bloomberg

Рассчитаны следующие переменные: ошибка прогноза на начало периода, ошибка прогноза на конец периода, пересмотр прогноза. Ошибка прогноза на начало периода - разница между реальным показателем и соответствующим прогнозом на начало периода, разделенная на реальный показатель. Ошибка прогноза на конец периода была рассчитана аналогичным образом. В качестве переменной пересмотра прогноза была рассмотрена разность между прогнозом на конец периода и прогнозом на начало периода, разделенная на прогноз на конец периода. Также была создана бинарная переменная, равная единице, если пересмотр прогноза был в сторону понижения, и равная нулю в любом другом случае. Для графического анализа была посчитана переменная, отвечающая за темп роста чистой прибыли по отношению к предыдущему году.

При анализе выборки был исключен ряд экстремальных значений переменных: удалены значения, входящие в первый и последний персентили соответствующих распределений, а также переменные, входящие в первые и последние пять персентилей, были винсоризованы ${ }^{2}$. Согласно описательным статистикам, в среднем на российском рынке наблюдались следующие тенденции. Период с 2005 по 2007 годы характеризовался тем, что и в конце, и в начале периода аналитики были оптимистичны, однако пересмотр прогноза к концу периода был в сторону понижения. Также в это время наблюдалась положительная динамика прибыли. Период с 2009 по 2012 год отличался от докризисного тем, что, несмотря на оптимизм в начале периода, к его концу аналитики были пессимистичны, что характеризуется положительной ошибкой прогноза. В кризис 2008 года компании в среднем продемонстрировали отрицательный прирост прибыли. Ожидания аналитиков в начале периода были завышенными, однако к его концу ошибка прогноза была близка к нулю. Для всех временных отрезков характерно уменьшение абсолютного значения ошибки прогноза к концу периода, а также уменьшение дисперсии ошибки. Это свидетельствует о том, что к концу года аналитики располагают большим количеством информации о деятельности компании, что позволяет им сделать более точные прогнозы.

1 *. Мультипликатор отношения балансовой стоимости компании к ее рыночной стоимости.

2*. Мультипликатор отношения рыночной стоимости компании к ее прибыли.

2. Винсоризация переменных в данном случае означает замену 5\% наибольших и 5\%наименьших переменных наибольшим и наименьшим значением из оставшегося массива данных, соответственно. 


\section{Результаты выявления использования рыночных ожиданий в управлении российскими компаниями}

Результаты графического анализа вылвления управления ожиданиями на российском рынке

Исследованы доли компаний, оправдавших ожидания аналитиков, и компаний, показавших положительный прирост прибыли по сравнению с предыдущим периодом. Сравнительная динамика данных показателей за 2005-2012 годы представлена на графике 1.

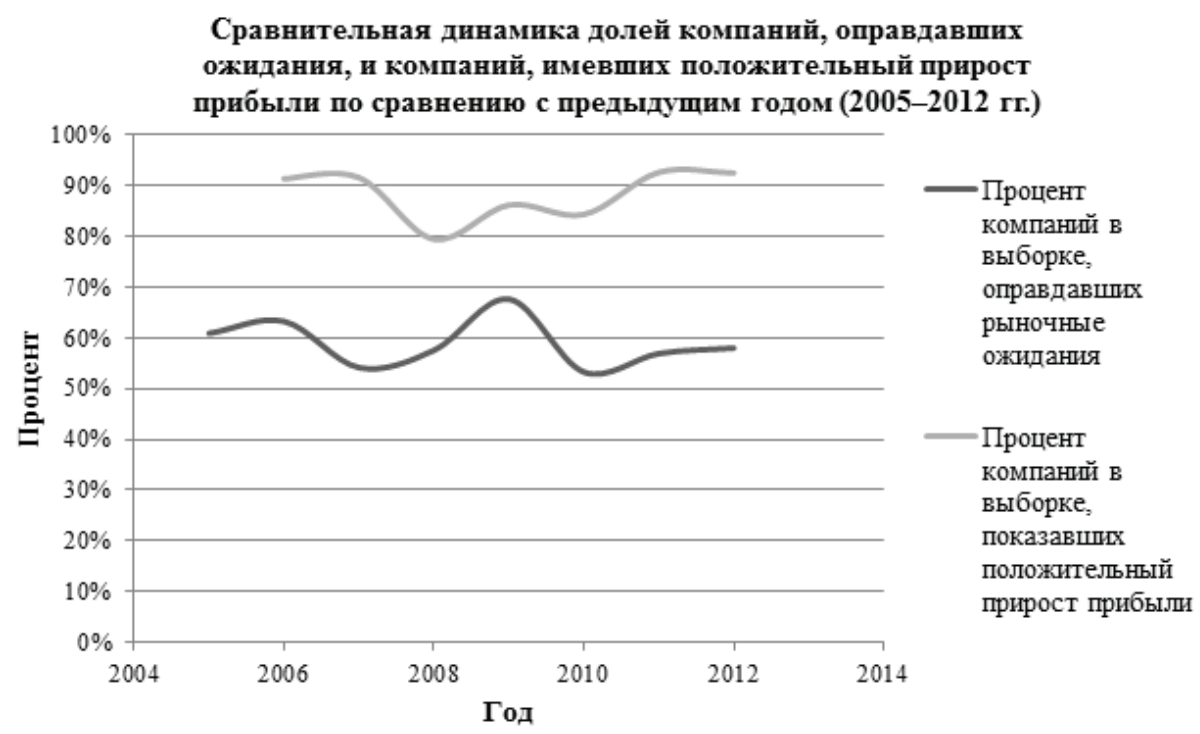

График 1. Сравнительная динамика долей компаний, оправдавших ожидания, и компаний, имевших положительный прирост прибыли по сравнению с предыдущим годом (2005-2012 г2.).

Источник: Рассчитано на основе данных Bloomberg.

В целом динамика доли компаний, оправдавших ожидания, повторяет динамику доли компаний, показавших положительный прирост чистой прибыли. Основное отличие заключается в том, что динамика доли компаний первой группы характеризуется более резкими падениями и подъемами. Это говорит о том, что данная доля объясняется чем-то еще помимо динамики прибыли. Примечательным является резкий рост доли компаний, соответствующих ожиданиям, в 2009 году, при том что рост доли компаний с положительным приростом прибыли не был столь стремительным. С одной стороны, это можно объяснить посткризисным пессимизмом аналитиков. С другой стороны, это может свидетельствовать о применении менеджерами управления ожиданиями либо управления прибылью. Примечательным является также тот факт, что, согласно описательным статистикам, пересмотр оптимистичных ожиданий в начале периода в сторону пессимизма к концу периода появился после кризиса 2008 года. Таким образом, на основе графического анализа и анализа средних показателей можно сделать вывод, что на российском рынке присутствуют определенные признаки управления ожиданиями, однако для более точных выводов необходим дальнейший анализ.

\section{Результаты анализа сопряженности признаков}

На следующем шаге исследована степень сопряженности следующих пар переменных: BGUIMGUI, BGUI-MEET, DOWN-MEET, MGUI-loss, BGUI-loss, refor_down-MEET. Переменная loss представляет собой бинарную переменную, равную единице, если фирма в текущем году показала убытки, и нулю в любом другом случае. Переменная refor_down также является бинарной переменной, равной единице в том случае, если аналитики пересмотрели свой прогноз по компании в сторону понижения, и нулю в любом другом случае. Соответствующие таблицы сопряженности были построены для всего рассматриваемого периода, для всего периода без кризисного 2008 года, для периода 2009-2012 годов и отдельно для 2010-2012 годов. Таблицы сопряженности не строились для докризисного периода 2005-2007 годов, так как в этом периоде было доступно недостаточное для анализа количество наблюдений 
(таблицы 2-5). Также не рассматривались таблицы сопряженности для пары переменных MGUI-MEET, так как переменная MGUI построена на основе MEET, что обеспечивает высокий коэффициент сопряженности для данной пары. В итоге значимая взаимосвязь была получена для пар переменных BGUI-MGUI, BGUI-MEET для всех периодов, а также для пары BGUI-loss за период 2005-2012 годов. Часть полученных таблиц со значимой взаимосвязью представлена ниже.

Согласно проведенному анализу сопряженности признаков, переменные MGUI и BGUI примерно одинаково отслеживают факт отсутствия управления ожиданиями, однако переменная BGUI ловит всего $27 \%$ случаев манипуляций со стороны менеджеров от случаев, пойманных переменной MGUI. Таким образом, эти две спецификации переменных не являются идентичными. Из таблицы 2 видно, что количество фирм, не использовавших управление ожиданиям и оправдавших прогнозы аналитиков, составляет 50\% от общего числа рассматриваемых компаний. При этом более 90\% компаний, использовавших управление ожиданиями, соответствовали прогнозам аналитиков. Данный факт соотносится с теорией управления ожиданиями и свидетельствует о довольно точном отображении этого феномена при помощи переменной BGUI. Также переменная BGUI показывает свою состоятельность при анализе сопряженности с переменной loss. Как показывают исследования, фирмы, несущие убытки, менее склонны управлять ожиданиями (Matsumoto, 2002). Анализ сопряженности BGUI и loss показывает, что только 7,46\% фирм, несущих убытки, использовали управление ожиданиями, что соотносится с выводами предыдущих работ. Результаты анализа сопряженности за другие периоды представлены в таблицах 3-5.

В общем и целом анализ сопряженности признаков показал, что переменные, выбранные для отслеживания управления ожиданиями на российском рынке, соотносятся с теоретическими предположениями относительно их поведения. Значимость и тип сопряженности переменных MGUI и BGUI, BGUI и MEET сохраняется для всех рассматриваемых временных отрезков. Более того, теоретическая состоятельность переменных увеличивает вероятность того, что на российском рынке присутствует управление ожиданиями.

Таблица 2

Результаты анализа сопряженности признаков за период 2005-2012 гг

\begin{tabular}{|c|c|c|c|}
\hline \multicolumn{4}{|c}{} \\
\hline \multirow{3}{*}{ MGUI } & & $\mathbf{0}$ & $\mathbf{1}$ \\
\cline { 2 - 4 } & $\mathbf{0}$ & $90,78 \%$ & $9,22 \%$ \\
\cline { 2 - 4 } & $\mathbf{1}$ & $72,73 \%$ & $27,27 \%$ \\
\hline
\end{tabular}

\begin{tabular}{|c|c|c|c|}
\hline \multicolumn{4}{|c|}{ MEET } \\
\hline & & $\mathbf{0}$ & $\mathbf{1}$ \\
\hline \multirow{2}{*}{ BGUI } & $\mathbf{0}$ & $49,12 \%$ & $50,88 \%$ \\
\cline { 2 - 4 } & $\mathbf{1}$ & $8.96 \%$ & $91,04 \%$ \\
\hline
\end{tabular}

\begin{tabular}{|c|c|}
\hline Всего наблюдений: & 272 \\
\hline \multicolumn{2}{|c|}{$\operatorname{chi} 2(1)=13,86 \operatorname{Pr}>$ chi $2=0,0002$} \\
\hline
\end{tabular}

\begin{tabular}{|c|c|}
\hline Всего наблюдений: & 578 \\
\hline \multicolumn{2}{|c|}{$\operatorname{chi} 2(1)=38,70 \operatorname{Pr}>\operatorname{chi} 2=0,000$} \\
\hline
\end{tabular}

\begin{tabular}{|c|c|c|c|}
\hline \multicolumn{4}{|c}{} \\
\multicolumn{2}{c|}{ Loss } \\
\hline \multirow{3}{*}{ BGUI } & & $\mathbf{0}$ & $\mathbf{1}$ \\
\cline { 2 - 4 } & $\mathbf{0}$ & $84,93 \%$ & $15,07 \%$ \\
\cline { 2 - 4 } & $\mathbf{1}$ & $92,54 \%$ & $7,46 \%$ \\
\hline
\end{tabular}

\begin{tabular}{|c|c|}
\hline Всего наблюдений: & 578 \\
\hline \multicolumn{2}{|c|}{$\operatorname{chi} 2(1)=2,81 \operatorname{Pr}>\operatorname{chi} 2=0,0934$} \\
\hline
\end{tabular}

Источник: Рассчитано на основе данных Bloomberg. 
Результаты анализа сопряженности признаков, 2005-2012 гг., за исключением 2008 г

\begin{tabular}{|c|c|c|c|}
\hline \multicolumn{4}{|c}{} \\
\hline \multirow{3}{*}{ MGUI } & & $\mathbf{0}$ & $\mathbf{1}$ \\
\cline { 2 - 4 } & $\mathbf{0}$ & $90,42 \%$ & $9,58 \%$ \\
\cline { 2 - 4 } & $\mathbf{1}$ & $73,02 \%$ & $26,98 \%$ \\
\hline \multicolumn{3}{|c|}{ Всего наблюдений: } & 230 \\
\hline \multicolumn{4}{|c|}{ chi2(1) $=11,27 \operatorname{Pr}>\operatorname{chi} 2=0,0008$} \\
\hline
\end{tabular}

\begin{tabular}{|c|c|c|c|}
\hline & & & MEET \\
\hline \multirow{3}{*}{ BGUI } & & $\mathbf{0}$ & 1 \\
\hline & $\mathbf{0}$ & $48,28 \%$ & $51,72 \%$ \\
\hline & 1 & $10,17 \%$ & $89,83 \%$ \\
\hline \multicolumn{3}{|c|}{ Всего наблюдений: } & 496 \\
\hline
\end{tabular}

Источник: рассчитано по данным Bloomberg.

Таблийа 4

Результаты анализа сопряженности признаков, 2009-2012 гг

\begin{tabular}{|c|c|c|c|}
\hline \multicolumn{2}{|c}{} & \multicolumn{2}{r|}{ BGUI } \\
\hline \multirow{3}{*}{ MGUI } & & $\mathbf{0}$ & $\mathbf{1}$ \\
\cline { 2 - 4 } & $\mathbf{0}$ & $89,73 \%$ & $10,27 \%$ \\
\cline { 2 - 4 } & $\mathbf{1}$ & $74,51 \%$ & $25,49 \%$ \\
\hline
\end{tabular}

\begin{tabular}{|c|c|c|c|}
\hline \multicolumn{4}{|c|}{ MEET } \\
\hline \multirow{3}{*}{ BGUI } & & $\mathbf{0}$ & $\mathbf{1}$ \\
\cline { 2 - 4 } & $\mathbf{0}$ & $48,58 \%$ & $51,42 \%$ \\
\cline { 2 - 4 } & $\mathbf{1}$ & $12,50 \%$ & $87,50 \%$ \\
\hline
\end{tabular}

\begin{tabular}{|l|c|}
\hline Всего наблюдений: & 197 \\
\hline \multicolumn{2}{|c|}{$\operatorname{chi} 2(1)=7,18 \operatorname{Pr}>\operatorname{chi} 2=0,0074$} \\
\hline
\end{tabular}

\begin{tabular}{|c|c|}
\hline Всего наблюдений: & 287 \\
\hline \multicolumn{2}{|c|}{$\operatorname{chi} 2(1)=18,23 \operatorname{Pr}>$ chi $2=0,0000$} \\
\hline \multicolumn{2}{|c|}{ Источник: рассчитано в по даннылм Bloomberg }
\end{tabular}

Таблица 5

Результаты анализа сопряженности признаков, 2010-2012 гг

\begin{tabular}{|c|c|c|c|}
\hline \multicolumn{2}{|c|}{} & \multicolumn{2}{r|}{ BGUI } \\
\hline \multirow{3}{*}{ MGUI } & & $\mathbf{0}$ & $\mathbf{1}$ \\
\cline { 2 - 4 } & $\mathbf{0}$ & $91,92 \%$ & $8,08 \%$ \\
\cline { 2 - 4 } & $\mathbf{1}$ & $78,72 \%$ & $21,28 \%$ \\
\hline
\end{tabular}

\begin{tabular}{|c|c|c|c|}
\hline \multicolumn{2}{|c|}{} & \multicolumn{2}{r|}{ MEET } \\
\hline \multirow{3}{*}{ BGUI } & & $\mathbf{0}$ & $\mathbf{1}$ \\
\cline { 2 - 4 } & $\mathbf{0}$ & $51,65 \%$ & $48,35 \%$ \\
\cline { 2 - 4 } & $\mathbf{1}$ & $10,34 \%$ & $89,66 \%$ \\
\hline
\end{tabular}

\begin{tabular}{|c|c|}
\hline Всего наблюдений: & 146 \\
\hline \multicolumn{2}{|c|}{$\operatorname{chi} 2(1)=5,13 \operatorname{Pr}>\operatorname{chi} 2=0,0235$} \\
\hline
\end{tabular}

\begin{tabular}{|c|c|}
\hline Всего наблюдений: & 211 \\
\hline \multicolumn{2}{|c|}{ chi2(1)=17,18 $\operatorname{Pr}>$ chi2 $2=0,0000$} \\
\hline \multicolumn{2}{|c|}{ Источник: рассчитано по даннылм Bloomberg } \\
\hline
\end{tabular}

Таким образом, на первом этапе исследования установлено, что управление ожиданиями присутствует в деятельности менеджмента российских публичных компаний. В наибольшей степени компании стали применять его после кризиса 2008 года. Наличие управления ожиданиями подтверждается тем, что прогнозы аналитиков на российском рынке не являются рациональными. Динамика доли компаний, оправдавших ожидания, не объясняется полностью динамикой прибыли. В наибольшей степени данное различие проявляется в посткризисный период.

В последующих частях исследования мы покажем гипотезы и результаты тестирования мотивов управления ожиданиями и характеристик российских компаний, следующей такой политике.

\section{Список использованной литературы}

1. Ивашковская И.В. Моделирование стоимости компании. Стратегическая роль советов директоров. М.: ИНФРА-М, 2009..

2. Athanasakou, V., Strong, N., Martin, W. (2011), The market reward for achieving analysts' earnings expectations: does managing expectations or earnings matter?, Journal of Business Finance and Accounting, 38(1) \& (2) (2011) 58-94.

3. Bartov, E., Givoly, D., Hayn, C. (2002), The reward to meeting or beating earnings expectations. Journal of Accounting and Economics, 33 (2002) 172-204. 
4. Bradshaw, M. (2011), Analysts' forecasts: what do we know after decades of work? URL: http:// ssrn.com/abstract=1880339.

5. Brown, L., Higgins, H. (2005), Managers' forecast guidance of analysts: International evidence, Journal of accounting and Public Policy, 24 (2005) 280-299.

6. Bulkley, G., and Harris, R. (1997), Irrational analysts' expectations as a cause of excess volatility in stock prices. The Economic Journal, 107 (1997) 359-371.

7. Copeland, T., Dolgoff, A., Moel, A. (2004), The role of expectations in explaining the cross-section of stock returns, Review of Accounting Studies, 9 (2004) 149-188.

8. Cotter, J., Tuna, I., Wysocki, P. (2006), Expectations management and beatable targets: how do analysts react to explicit earnings guidance?. Contemporary Accounting Research, 3(23) (2006) 593-624.

9. Koh, K., Matsumoto, D., Rajgopal, S. (2008), Meeting or beating analyst expectations in the postscandals world: changes in stock market rewards and managerial actions, Contemporary Accounting Research, 4(25) (2008) 1067-1098.

10. La Porta, R. (1996), Expectations and the cross-section of stock returns, The Journal of Finance, 5(LI) (1996) 1715-1742.

11. Matsumoto, D. (2002). Management's incentives to avoid negative earnings surprises. The Accounting Review, 3(77) (2002) 483-514.

12. Sankaraguruswamy, S., Sweeney, R. (2005), Earnings management and expectations management: implications for analyst rationality. URL: http://papers.ssrn.com/sol3/papers.cfm?abstract $\underline{\mathrm{id}=683041}$. 